\title{
Abnormal Reward System Activation in Mania
}

\author{
Birgit Abler*,', Ian Greenhouse ${ }^{2}$, Dost Ongur ${ }^{3}$, Henrik Walter ${ }^{4}$ and Stephan Heckers ${ }^{5}$ \\ 'Department of Psychiatry University of Ulm, Germany; ${ }^{2}$ University of California, San Francisco, CA, USA; ${ }^{3}$ McLean Hospital, Harvard University, \\ Belmont, MA, USA; ${ }^{4}$ Department of Psychiatry, Division of Medical Psychology, University of Bonn, Germany; ${ }^{5}$ Department of Psychiatry, \\ Vanderbilt University, Nashville, TN, USA
}

\begin{abstract}
Transmission of reward signals is a function of dopamine, a neurotransmitter known to be involved in the mechanism of psychosis. Using functional magnetic resonance imaging ( $\mathrm{fMRI}$ ), we investigated how expectation and receipt of monetary rewards modulate brain activation in patients with bipolar mania and schizophrenia. We studied 12 acutely manic patients with a history of bipolar disorder, 12 patients with a current episode of schizoaffective disorder or schizophrenia and 12 healthy subjects. All patients were treated with dopamine antagonists at the time of the study. Subjects performed a delayed incentive paradigm with monetary reward in the scanner that allowed for investigating effects of expectation, receipt, and omission of rewards. Patients with schizophrenia and healthy control subjects showed the expected activation of dopaminergic brain areas, that is, ventral tegmentum activation upon expectation of monetary rewards and nucleus accumbens activation during receipt vs omission of rewards. In manic patients, however, we did not find a similar pattern of brain activation and the differential signal in the nucleus accumbens upon receipt vs omission of rewards was significantly lower compared to the healthy control subjects. Our findings provide evidence for abnormal function of the dopamine system during receipt or omission of expected rewards in bipolar disorder. These deficits in prediction error processing in acute mania may help to explain symptoms of disinhibition and abnormal goal pursuit regulation.

Neuropsychopharmacology (2008) 33, 22 17-2227; doi:I 0. I038/sj.npp. I 301620; published online 7 November 2007
\end{abstract}

Keywords: $\mathrm{fMRI}$; mania; schizophrenia; reward; dopamine system; nucleus accumbens

\section{INTRODUCTION}

Mania has been characterized as a disorder of abnormal goal pursuit regulation with elevated levels of achievement motivation and drive. Patients with bipolar mania tend toward high goal setting and unrealistically high success expectancies (Johnson, 2005). Besides an increase of goaldirected activity they show excessive involvement in pleasurable activities that have a high potential for 'painful consequences, eg unrestrained buying sprees or foolish business investments' (DSM-IV, 2000).

A dysregulation of reward-related neural networks mediating motivation and goal-directed behavior was suggested to account for these observations (Miller, 1993; Johnson, 2005). Deficits in performing response-reversal tasks, modeling behavioral adaptation to changing reward contingencies in euthymic children with bipolar disorder, support this notion (Gorrindo et al, 2005). Further, it has been shown that induction of happy mood with a reward

\footnotetext{
*Correspondence: Dr B Abler, Department of Psychiatry, University of Ulm, Leimgrubenweg 12-14, Ulm BW 89075, Germany, Tel. + 49073150061551 , Fax: + 49073150021549 , E-mail: birgit.abler@uni-ulm.de

Received 4 June 2007; revised 30 September 2007; accepted 3 October 2007
}

task results in prolonged elevated mood in euthymic bipolar patients compared to healthy controls (Farmer et al, 2006).

Functional magnetic resonance imaging (fMRI) studies have revealed prefrontal dysfunction in bipolar disorder and manic psychosis, but evidence for abnormalities in reward-related neural network function in mania is scarce (Yurgelun-Todd and Ross, 2006). One fMRI study has reported elevated amygdala activation in acute mania (Altshuler et al, 2005) and several studies have suggested alterations in the shape (Hwang et al, 2006), size (Strakowski et al, 2002), and function (Strakowski et al, 2005) of the basal ganglia in bipolar disorder.

A compelling reason to study the function of the mesolimbic dopaminergic system, including ventral tegmental area and nucleus accumbens (NAcc), in mania is the effectiveness of antipsychotic drugs in the treatment of acute mania and the prevention of relapses into manic episodes by neuroleptic medication (Perlis et al, 2006; Scherk et al, 2007). There is evidence that the dopamineantagonistic properties of these drugs are crucial at least for the acute effects (Nolen, 1983).

The clear link between activation of the dopaminergic mesolimbic system and reward functions (Abler et al, 2005, 2006; Knutson and Cooper, 2005; Knutson et al, 2001b) led us to study this system in mania. Besides anticipatory dopamine signals, we were interested in prediction error signals mediated by dopamine upon receipt and omission of 
reward (Abler et al, 2005, 2006). As the symptoms of untreated acute mania (including hyperactivity, excitement, and impaired decision-making capability) are not easily compatible with an fMRI study, we decided to investigate only medicated patients. In addition to a healthy control group, we also studied a disease control group of patients with an acute episode of schizophrenia or schizoaffective disorder. Both patient groups were treated with comparable dosages of antipsychotic medication, providing the opportunity to disentangle the effects of medication and psychiatric diagnosis.

We designed this study to test two hypotheses: (1) Medicated bipolar patients during an acute manic episode will show deficient reward processing (Miller, 1993; Johnson, 2005; Gorrindo et al, 2005; Farmer et al, 2006) in the form of decreased reward-related brain activation. (2) Medicated patients during an acute episode of schizophrenia or schizoaffective disorder will not show similarly decreased reward-related brain activation (Juckel et al, 2006b), which provides evidence that the anticipated changes in medicated, manic patients are not simply due to an acute psychiatric illness or due to treatment with neuroleptic medication.

\section{MATERIALS AND METHODS}

\section{Subjects}

A total of 12 patients with a diagnosis of bipolar manic disorder (referred to as 'mania' in the following) admitted to McLean Hospital for an acute manic or mixed episode and one outpatient with a recent episode were included and completed the study. All bipolar subjects carried a diagnosis of Bipolar Disorder type I, eight of them were currently suffering from a manic, three from a mixed and one subject from a hypomanic episode. During the current episode, six of the bipolar patients included experienced moodcongruent, three patients mood-incongruent psychotic features. They were compared with 12 patients with a diagnosis of schizophrenia or schizoaffective disorder (referred to as 'schizophrenia' in the following) admitted for an acute episode of the illness to McLean Hospital and with 12

Table I Demographic and Clinical Characteristics of the Three Study Groups

\begin{tabular}{|c|c|c|c|c|c|c|}
\hline & $\begin{array}{c}\text { Patients-BPm } \\
(n=12) \text { Mean (SD) }\end{array}$ & $\begin{array}{c}\text { Patients -SZ } \\
(n=I 2) \text { Mean (SD) }\end{array}$ & $\begin{array}{c}\text { Controls-C } \\
(n=12) \text { Mean (SD) }\end{array}$ & $\begin{array}{l}\text { BPm vs SZ } \\
t(d f=22) ; p\end{array}$ & $\begin{array}{l}\text { BPm vs C } \\
t(d f=22) ; p\end{array}$ & $\begin{array}{c}\text { SZ vs C } \\
t(d f=22) ; p\end{array}$ \\
\hline Age (years) & $33.9(11.2)$ & $36.7(7.8)$ & $36.2(11.2)$ & $-0.70 ; 0.49$ & $-0.49 ; 0.63$ & $0.13 ; 0.90$ \\
\hline Male/female & $7 / 5$ & $5 / 7$ & $7 / 5$ & & & \\
\hline Education (years) & $15.7(1.9)$ & $13.8(1.5)$ & $15.3(2.2)$ & $2.67 ; 0.014^{*}$ & $0.5 ; 0.63$ & $-1.84 ; 0.081$ \\
\hline Novelty seeking & $20.9(7.8)$ & $20.3(4.4)$ & $17.2(5.15)$ & $0.22 ; 0.83$ & $1.39 ; 0.18$ & $1.61 ; 0.15$ \\
\hline Physical anhedonia & $13(8.5)$ & $15(3.5)$ & $12(6.6)$ & $-0.94 ; 0.38$ & $0.11 ; 0.92$ & I.3; 0.23 \\
\hline Social anhedonia & $7.7(6.1)$ & $15(6.7)$ & $10.7(6.7)$ & $-2.81 ; 0.026 *$ & $-1.15 ; 0.26$ & 1.58; 1.18 \\
\hline $\begin{array}{l}\text { Medication (mg of } \\
\text { CLPZ-equivalents) }\end{array}$ & $375(397)$ & $595(357)$ & - & $-1.43 ; 0.17$ & & \\
\hline $\begin{array}{l}\text { Total (positive, negative } \\
\text { general) }\end{array}$ & $62.0(23.0)$ & $74(16.9)$ & - & $-1.46 ; 0.16$ & & \\
\hline $\begin{array}{l}\text { Psychotic symptoms } \\
(\mathrm{PI}, \mathrm{P} 2, \mathrm{P} 3)\end{array}$ & $8.6(4.2)$ & $11.6(2.2)$ & - & $-2.21 ; 0.042 *$ & & \\
\hline $\begin{array}{l}\text { Brief psychiatric rating } \\
\text { scale }\end{array}$ & $31.9(14.5)$ & $35.7(10.3)$ & - & $-0.73 ; 0.47$ & & \\
\hline $\begin{array}{l}\text { Montgomery-Asberg } \\
\text { depression scale }\end{array}$ & II.8 (I5.4) & $13(9.6)$ & - & $-0.22 ; 0.83$ & & \\
\hline Young mania rating scale & $21.8(9.0)$ & $12.3(\mid 1.1)$ & - & $2.30 ; 0.032 *$ & & \\
\hline Years of illness & $12.8(\mid 1.7)$ & $15.6(5.2)$ & - & $-0.74 ; 0.47$ & & \\
\hline
\end{tabular}

Abbreviations: a, Asian; b, Black; BPm, bipolar manic disorder; C, Healthy Controls; c, Caucasian; NAART, North American Adult Reading Test; PANSS, Positive and Negative Symptom Scale; SD, standard deviation; SZ, schizophrenia/schizoaffective disorder; y, years.

${ }^{a}$ Not known for two schizophrenic and three bipolar patients; Psychotic symptoms (PI, P2, P3): Averaged scores of the sum of the three PANSS items characterizing psychotic symptoms: delusions, hallucinatory behavior, and conceptual disorganization.

Bold value significant at $P<0.05$. 
healthy control subjects (Table 1). Diagnoses were assessed according to the Diagnostic and Statistical Manual of Mental Disorders with a Structured Clinical Interview for DSM-IV (SCID) by a psychiatrist not involved in the neuroimaging study. None of the subjects had a history of major medical or neurological illness, and no healthy control subject had a history of psychiatric illness according to the SCID Axis I Disorders. All patients were taking a stable dose of antipsychotic medication. Three patients with schizophrenia and one bipolar manic patient were treated with first-generation antipsychotic medication. All other patients were on second-generation antipsychotic medication. Medication in the two patient groups (Table 1) did not differ in chlorpromazine equivalents received (Baldessarini and Tarazi, 2001).

All bipolar manic and eight of the patients with schizophrenia were on mood stabilizers (lithium, antiepileptic drugs). Seven patients with schizophrenia but no bipolar patient took antidepressant medication. Three schizophrenia patients and five bipolar patients received benzodiazepines (up to $5 \mathrm{mg}$ of clonazepam and $2 \mathrm{mg}$ of lorazepam per day). No subject met DSM-IV criteria for alcohol or substance abuse within the previous 6 months. No subject reported a history of alcohol or substance dependence. Several participants denied any use of illegal drugs (mania: six, schizophrenia: four, controls: six), some reported occasional use of cannabis, 10 or more years ago (mania: three, schizophrenia: four, controls: three) and some reported occasional cannabis use within the past 10 years (mania: three, schizophrenia: four, controls: three). Five of the patients with schizophrenia, but none of the bipolar patients and none of the controls reported having tried other illegal drugs (cocaine, opioids, LSD, mescaline). Some of the patients (mania: two, schizophrenia: seven) but none of the controls were cigarette smokers. Nine of the manic patients were hospitalized for three or less times, three patients for more than five times. Six of the patients with schizophrenia reported more than 10 hospitalizations in the past, the others at least two. Two of the bipolar manic and none of the patients with schizophrenia had a documented depressive episode in the past. Patients and controls did not differ significantly in age or level of parental attained formal education (Table 1). Manic subjects had significantly, and controls a trend toward, more years of attained education compared to patients with schizophrenia. Controls displayed a lower number of errors in the North American Adult Reading Test (NAART) than patients with schizophrenia.

All participants, patients and controls, gave written informed consent after complete description of the study. The study was carried out in accordance with the latest version of the Declaration of Helsinki and approved by the ethics committee of McLean Hospital, Belmont, MA.

\section{Ratings and Psychological Testing}

In addition to the SCID interview, we assessed all patients with the Positive and Negative Symptom Scale (PANSS), Brief Psychiatric Rating Scale, Montgomery Asberg Depression Rating Scale, and the Young Mania Rating Scale (YMRS).
After scanning, patients and healthy subjects were asked to complete two questionnaires: the Novelty Seeking subscale of Cloninger's Temperament and Character Inventory (Cloninger, 1987; Cloninger and Svrakic, 1997) and the BBPSA to assess scores of social and physical anhedonia. Outside of the scanner, subjects performed two additional simple reaction time tasks ( $\mathrm{RT} 1$ : simple reaction to visual stimulus, RT2: reaction to visual stimulus after audible warning tone).

\section{Reward Task}

We employed a previously validated monetary incentive task that parametrically modulated three possible wins (\$1.25\$0.40, no win) (Abler et al, 2005). Subjects completed two scanning sessions of 60 trials each (10 no-win-trials, 25 trials with potential gain of $\$ 1.25$ and 25 trials with potential gain of $\$ 0.40$ ). Each trial started with one of three symbols (cue, $750 \mathrm{~ms}$ ) indicating the possible amount of money to win. After an expectation period (delay, $3000 \mathrm{~ms}$ ), subjects had to correctly react with a left or right button press to one of two symbols (a square or a triangle; target) within a fixed interval of $1500 \mathrm{~ms}$ with the index or middle finger of their dominant (left or right) hand. Subjects were informed that their chances to win were independent of their reaction times. In reacting correctly, they preserved themselves a $60 \%$ chance to win the announced amount of money $(\$ 1.25$ or $\$ 0.40$ : win trial). In $40 \%$ of the trials, subjects were not rewarded despite pressing the correct button (omission trial). Incorrect button presses resulted in a feedback of zero dollars at any rate. Win and omission trials as well as the three trial types $(\$ 1.25, \$ 0.40$, no win) appeared in a random order. In the control trials (no win) no money was announced, subjects only had to press an arbitrary button and could not win any money. To make sure that all trials included a button press of any kind, subjects were informed that they would lose $\$ 1$ if no button press occurred. Feedback (outcome, $1500 \mathrm{~ms}$ ) followed the targets disappearance and notified subjects the amount of money they won in the trial. Reaction times and errors were registered. Median reaction times were calculated across trials for each single subject, means were calculated to average over subjects.

\section{fMRI Acquisition}

A 3 Tesla Siemens TRIO Scanner (Siemens AG, Germany) equipped with a head coil was used to acquire T1 anatomical volume images $(1 \times 1 \times 1 \mathrm{~mm}$ voxels $)$ and functional $M R$ images. A total of 21 axial slices were acquired with an image size of $64 \times 64$ pixels and a FoV of $192 \mathrm{~mm}$. Slice thickness was $3 \mathrm{~mm}$ with $0.75 \mathrm{~mm}$ gap resulting in a voxel size of $3 \times 3 \times 3.75 \mathrm{~mm}$. Images were angled along a line connecting basal forebrain and basal cerebellum. Images were centred on basal structures of the brain including subcortical regions of interest (basal ganglia, orbitofrontal, and ventral frontal regions). Functional images were recorded using a $\mathrm{T} 2{ }^{*}$-sensitive gradient echo sequence measuring changes in BOLD-contrast. A total of 426 volumes were obtained during each of the two reward sessions at a TR of $1500 \mathrm{~ms}$ (TE $35 \mathrm{~ms}$, flip 90 ). 


\section{fMRI Analysis}

Image processing and statistical analysis were carried out using Statistical Parametric Mapping (SPM2, Wellcome Department, London, UK). Images were pre-processed including slice timing, realignment to correct for motion artifacts, and spatial normalization to a standard template (Montreal Neurological Institute, MNI) with a resampled voxel size of $3 \times 3 \times 3 \mathrm{~mm}$. Smoothing was applied with an $8 \mathrm{~mm}$ Gaussian kernel. Intrinsic autocorrelations were accounted for by $\mathrm{AR}(1)$ and low frequency drifts were removed via high pass filter.

After preprocessing, first level analysis was performed on each subject estimating the variance of voxels according to a general linear model. The three expectation periods (including presentation of the cue) as well as the button press and the three different outcome events (win, omission, control trial) were each modeled as a boxcar function and convolved with the hemodynamic response function resulting in seven orthogonal regressors. The 6 realignment parameters were included in the model, resulting in 13 regressors. Contrast images for regressors of interest were calculated for each subject. The contrast images of the parameter estimates were then included in a second level group analysis (mixed effects model), treating subject as a random effect. One-sample $t$-tests were used to investigate within group effects and effects for all subjects together, two-sample $t$-tests for effects between groups. Statistical maps were thresholded at $p<0.001$ for the voxelwise analysis comprising all subjects, and $p<0.005$ for all other voxel-wise within and between group comparisons. In a priori ROI of the mesolimbic reward system (ie the ventral striatum (Abler et al, 2005, 2006; Knutson and Cooper, 2005; Knutson et al, 2001b) and the tegmental area/brain stem (Dreher et al, 2006)) the $p$-values were corrected for the number of comparisons made within each region using the small volume correction procedure (Worsley et al, 1996). A priori volumes of interest were derived from a previous pharmaco-fMRI experiment in healthy subjects with the same task from the trials without medication (Abler et al, 2007). A $10 \mathrm{~mm}$ sphere was centered in the voxels of maximal activation as found in this prior experiment (ventral striatum: $x / y / z=-6 / 15 /-3$, left or $9 / 15 / 0$, right; brain stem: $x / y / z=-12 /-24 /-21$, left or $12 /-24 /-15$, right; ventral tegmental area: $x / y / z=9 /-15 /-15$, right). As no left-sided ventral tegmental peak activation was found in our previous experiment, the right-sided activation was mirrored to the left for the volume of interest definition $(x / y / z=-9 /-15 /-15)$.

For the analysis of the signal time course data, functional ROIs were defined from the activations found in the analysis including all 36 subjects. For ROI definition, the following maps were thresholded at $p<0.005$ false discovery rate corrected to ensure that only voxels with strong effects were included: mania/schizophrenia/controls: expectation of high $>$ no reward and mania/schizophrenia/controls: outcome: win>omission. For each subject, the averaged, mean-corrected first eigenvariate of the signal intensity of all voxels within a predefined region was extracted to obtain fMRI signal time series. The event-related time courses as depicted in Figures 3 and 4 were obtained by first extracting series of 13 time points (TRs) from onset of each trial on and then averaging over all trials of one type, the two runs and the respective subjects. $T$-tests were used to compute differential effects in the ROI time series using external software (Microsoft: Excel, Statsoft: Statistica).

Between group effects within the ROIs were calculated using MarsBar (Brett et al, 2002).

\section{RESULTS}

\section{Clinical Rating Scales}

Schizophrenia patients scored higher on the Social Anhedonia and the Negative Syndrome Scale and lower on the YMRS compared with bipolar patients (Table 1).

To further characterize the level of psychosis, we calculated the average scores of the sum of the PANSS items for delusions, hallucinatory behavior, and conceptual disorganization. We found significantly higher levels in the schizophrenia group than in the bipolar manic patients. The mean YMRS score of 21.8 in the bipolar group indicates a significant level of manic symptoms, similar to previous studies of cognitive function in acute mania (Blumberg et al, 2003; Clark et al, 2001).

\section{Accuracy and Reaction Time Data}

The mean accuracy and reaction time for all trials and all three groups were $96.5 \%$ and $768 \mathrm{~ms}$, respectively. Both patient groups were less accurate (mania: 95.7\%; $t(11)=2.79 ; p<0.01$; schizophrenia: $94.9 \% ; t(11)=2.85$; $p<0.01$ ) and slower (mania: $829 \mathrm{~ms}$, SD 99; $t(11)=3.91$; $p<0.001$ schizophrenia: $768 \mathrm{~ms}, \quad$ SD $91 ; t(11)=2.12$; $p<0.05)$ than the control group $(99.0 \%$ and $705 \mathrm{~ms}, \mathrm{SD}$ $47)$. The two patient groups did not differ significantly in accuracy or reaction time.

As expected (Abler et al, 2005, 2006), reaction times in the controls (no/low/high reward: 715/718/684 ms) were significantly faster in the high reward trials than in the low $(p<0.005)$ or the no reward trials $(p>0.05)$ (Figure 1$)$. This main effect of reward was seen in the schizophrenia group as well (no/low/high reward: 780/778/746 ms; $p<0.03$ for

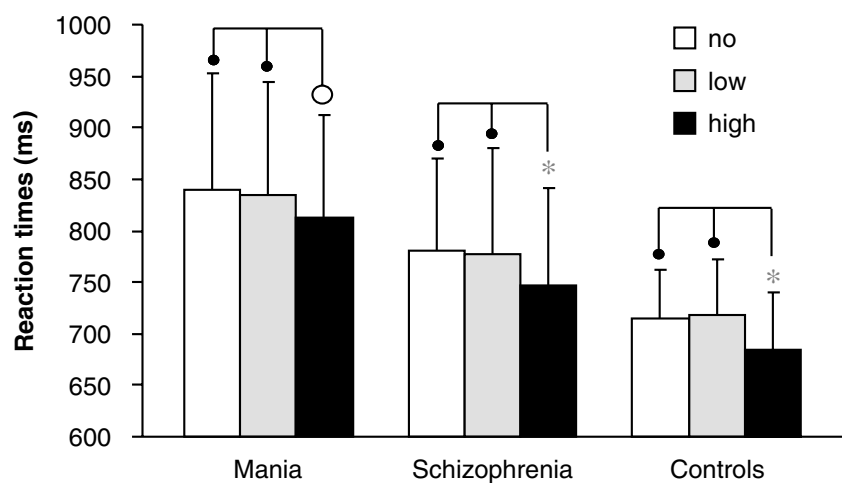

Figure I Mean reaction times and standard deviation during scanning of the reward task in the three groups (for the three trial types: high/low/no reward). In the high reward trials of the controls and SZ patient groups, we observed a significant $(* p<0.05)$ acceleration of reaction times compared to both the low and no reward trials, which was not present $(O)$ in the manic patients. 
Table 2 Brain activation in a priori regions of interest (ventral striatum, tegmental area/brain stem) during the expectation phase (high $>$ no reward and high $>$ low reward) and the outcome phase (win > omission) of the experiment

\begin{tabular}{|c|c|c|c|c|c|c|c|c|c|c|c|c|c|c|c|}
\hline \multirow[b]{2}{*}{ Contrast/region } & \multicolumn{4}{|c|}{ Overall } & \multicolumn{3}{|c|}{ Controls, C } & \multicolumn{3}{|c|}{ Schizophrenia, S } & \multirow[t]{2}{*}{ Mania, M } & \multicolumn{2}{|c|}{ C vs $M$} & \multirow[t]{2}{*}{ C vs S } & \multirow[t]{2}{*}{$S$ vs $M$} \\
\hline & $L / R$ & Z & NV & $\begin{array}{l}\text { Peak coordinates } \\
\qquad / y / z\end{array}$ & Z & NV & $\begin{array}{c}\text { Peak coordinates } \\
x / y / z\end{array}$ & Z & NV & $\begin{array}{c}\text { Peak coordinates } \\
x / y / z\end{array}$ & & NV & $\begin{array}{c}\text { Peak coordinates } \\
x / y / z\end{array}$ & & \\
\hline Expectation: high > no reward & & & & & & & & & & & NS & & NS & NS & NS \\
\hline \multirow[t]{2}{*}{ Ventral tegmental area } & $\mathrm{R}$ & 6.88 & 950 & $9 /-12 /-12$ & 7.10 & 441 & $6 /-12 /-12$ & 5.72 & 47 & $6 /-12 /-15$ & & & & & \\
\hline & $L$ & 5.68 & a & $-6 /-12 /-15$ & 6.50 & a & $-3 /-9 /-12$ & & & & & & & & \\
\hline \multirow[t]{2}{*}{ Nucleus accumbens } & $\mathrm{R}$ & 5.22 & a & $12 / 3 /-6$ & 6.01 & a & $9 / 6 / 0$ & 4.28 & 83 & |8/6/-9\# & & & & & \\
\hline & L & 4.48 & a & $-12 / 9 /-3$ & 3.80 & a & $-9 / 6 /-3 \#$ & & & & & & & & \\
\hline Expectation: high > low reward & & & & & & & & & & & NS & & NS & NS & NS \\
\hline \multirow[t]{2}{*}{ Brainstem } & $\mathrm{R}$ & 4.63 & 79 & $6 /-24 /-12$ & 4.72 & b & $6 /-24 /-18$ & & & & & & & & \\
\hline & $L$ & 4.37 & a & $-3 /-27 /-12$ & 4.85 & 85 & $-6 /-36 /-18$ & & & & & & & & \\
\hline Outcome: win > omission & & & & & & & & & & & NS & & & NS & NS \\
\hline \multirow[t]{2}{*}{ Nucleus accumbens } & $\mathrm{R}$ & 6.62 & 252 & $9 / 12 /-6$ & & & & 5.27 & 71 & $6 / 9 /-6$ & & & & & \\
\hline & $L$ & 5.05 & a & $-9 / 9 /-6$ & 6.64 & 294 & $-6 / 9 /-6$ & & & & 3.98 & 24 & $-3 / 12 /-9$ & & \\
\hline \multicolumn{16}{|c|}{$\begin{array}{l}\text { Abbreviations: BA, Brodmann area; IFG: inferior frontal gyrus; L/R/M, left/right/midline; } \\
\text { small volume correction; Z, Z-level at peak coordinate. } \\
\text { Significant activations are listed for an overall analysis of all three groups together (first } \\
\text { a level of } p<0.05 \text { corrected for the number of comparisons made within each regic } \\
\text { Coordinates are SPM/MNI-coordinates. } \\
\text { NS, no significant differences for this contrast at the given level. } \\
\text { \#Significance level } p<0.005 \text { (voxel level) and } p<0.05 \text { (cluster level) uncorrected. } \\
\text { aPart of cluster above. } \\
\text { bPart of cluster below. }\end{array}$} \\
\hline
\end{tabular}


low and $p<0.01$ for no reward trials), but was attenuated and not significant in the manic group (no/low/high: 839/ $835 / 812 \mathrm{~ms}$ ). A $2 \times 3$ ANOVA with the factors 'group' and 'condition' did not reveal significant interaction effects $(\mathrm{F}=0.13, p=0.97)$.

Average reaction times in the tests after scanning (RT1/ RT2) were significantly faster in the controls group (302/ $268 \mathrm{~ms})$ than in schizophrenia (386/356 ms; $p=0.0005 /$ $0.001)$ or manic patients $(404 / 380 \mathrm{~ms}, p=0.0008 / 0.0009)$. Performance in the patient groups was not significantly different for the two tasks $(p=0.43 / 0.33)$.

\section{Functional Imaging Data}

We investigated main effects of group (mania, schizophrenia, and controls) and group-by-condition interactions to study the two phases of the experiment: the expectation phase (contrast (1) expectation of high $v s$ no reward, contrast (2) expectation of high $v s$ low reward) and the outcome phase (contrast (3) win vs omission of reward, contrast (4) omission of reward vs win) (Abler et al, 2005). Out of these, the contrasts $1-3$ yielded meaningful results at the predefined thresholds and are reported here. Table 2 shows the results in a priori ROI (ventral striatum, tegmental area/brain stem).

We were able to replicate previous findings in an overall analysis of all three groups. We found activation of NAcc, ventral tegmental area, anterior insula, parahippocampus, cingulate, and medial prefrontal cortex for contrast 1 (expectation of high vs no reward) and activation of anterior insula/inferior frontal gyrus and brain stem in contrast 2 (expectation of high vs low reward) analysis. Contrast 3 (win $v s$ omission of reward) showed activation of NAcc, cingulate and medial prefrontal cortex, and the right parahippocampus.

This pattern of reward-related brain regions was similar but less pronounced in the analysis of the 12 control subjects (Table 2), with ventral tegmental area and bilateral ventral striatum activation upon expectation of high versus no reward (contrast 1) and NAcc and medial prefrontal cortex activation upon win versus omission of reward (contrast 3). Likewise, we found ventral tegmental and NAcc activation upon expectation of high $v s$ no reward and NAcc activation upon receipt versus omission of reward in the analysis of the 12 subjects with schizophrenia. In contrast, we did not find any activation of the reward circuitry in the analysis of the 12 manic subjects.

To confirm the finding of decreased reward circuitry activation in the bipolar subjects, we tested for significant group-by-condition interactions in contrasts 1 and 3, using the control group activation maps as an inclusive mask (voxel-wise comparison, thresholded at $p<0.005$ at the voxel- and $p<0.05$ at the cluster level). This analysis revealed a significantly greater activation in the left NAcc $(x / y / z=-3 / 12 /-9, Z=3.98 \mathrm{NV}=24$, Figure 2) in healthy control compared with bipolar manic subjects. No between group effects were found when comparing the two patient groups.

To further investigate the fMRI signal in our groups, we defined functional ROIs in the main activation clusters from the analyses of all 36 subjects: left and right ventral tegmental area activation during the expectation phase
(Figure 3) and left and right NAcc activation during the outcome phase (Figure 4). Using MarsBAR, we calculated group differences for the brain activation within these ROIs. We found no significant group differences of ventral tegmental area activation during the expectation phase (Figure 3) but did find greater activation of the control subjects compared with the bipolar subjects during the outcome phase in left NAcc $(t=2.42 ; p=0.012)$ and right NAcc $(t=1.56 ; p=0.066)$ (Figure 4$)$.

We then extracted signal time courses from left and right ventral tegmental area (Figure 3 ) and from left and right NAcc (Figure 4) separately for each group. Calculating activation differences (high $v s$ no reward) from the signal time courses of the VTA for scans 5-8 as depicted in the time course graphs in Figure 3 (corresponding to the expectation phase), we found significant differences in the time courses of the control and schizophrenia group but not the mania group (Figure 3). Signal time courses from the NAcc showed significant differences between receipt and omission of reward (approximately corresponding to scan 9 and 10$)$ only in the control group and a trend $(p=0.078)$ in the schizophrenia group (right NAcc).

Differences between receipt and omission of reward in the signal time courses were greater in the control subjects as compared to the bipolar manic subjects (Controls $>$ Mania [win $>$ omission]) in both, right and left NAcc (Figure 4).

\section{DISCUSSION}

Our study provides the expected evidence for neural correlates of altered reward processing in bipolar manic patients. Mere medication effects could be excluded as both, healthy controls and medicated patients with schizophrenia showed elevated activation of dopaminergic brain areas when expecting high rewards compared to anticipation of no rewards, which is in line with previous studies of healthy volunteers (Abler et al, 2005; Knutson et al, 2001a). We also replicated the previously reported pattern of decreased

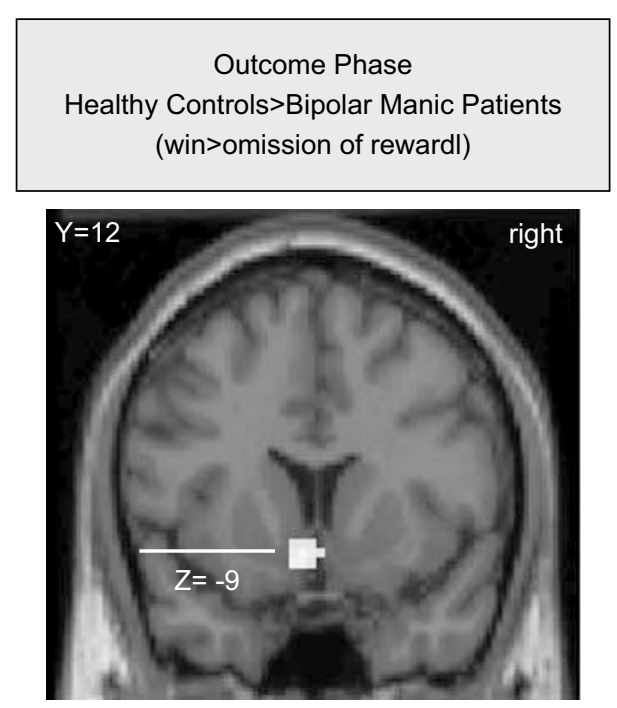

Figure 2 Differential $\mathrm{fMRI}$ activation (controls (outcome: win $>$ omission of reward) > mania (outcome: win $>$ omission of reward)) at $p<0.005$, uncorrected for multiple comparisons. 

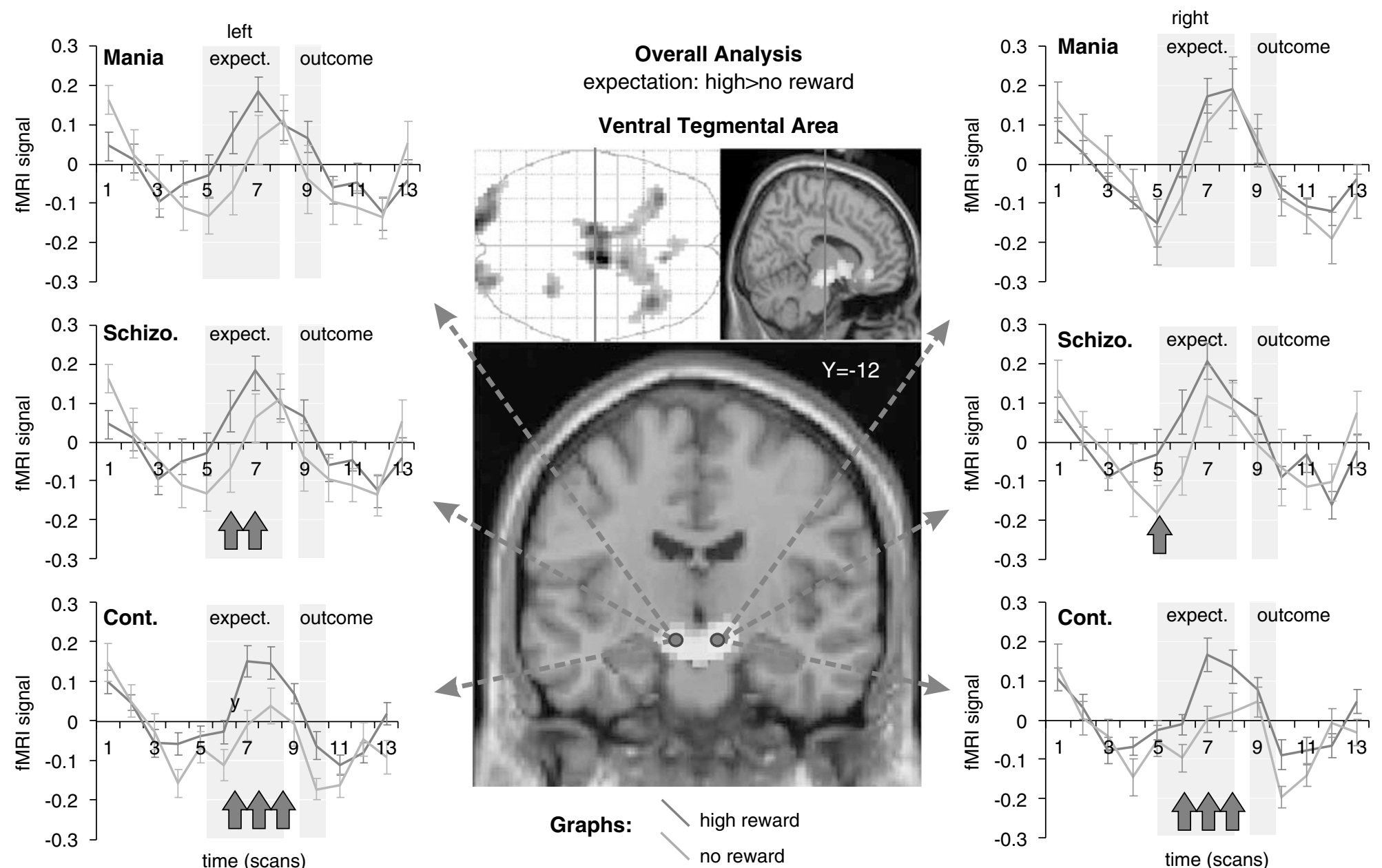

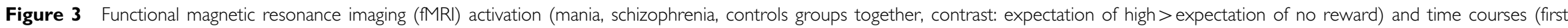
elgenvariate of the fMRI signal intensity as provided by standard SPM functions) in left and right ventral tegmental area for each of the groups. The map was thresholded at $p<0.00$ land extent threshold

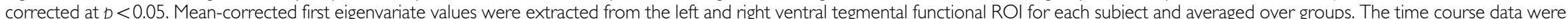
averaged event-related to depict the mean fMRI signal and standard error related to expectation of high and no reward in each of the three groups. Grey shades indicate the period when reward

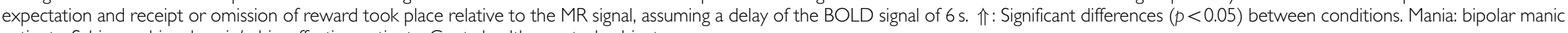
patients; Schizo.: schizophrenia/schizoaffective patients; Cont.: healthy control subjects. 

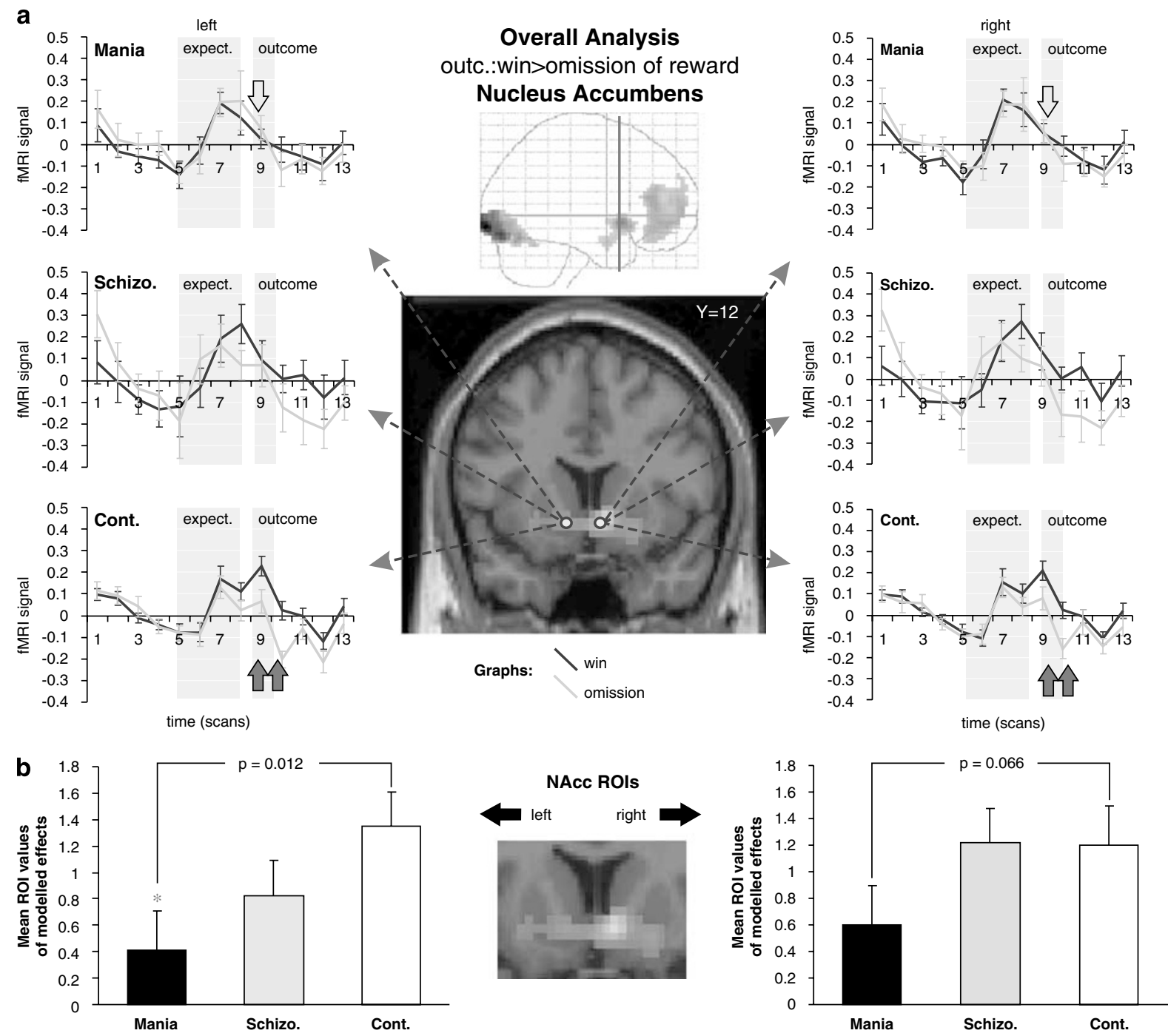

Figure 4 (a) Differential functional magnetic resonance imaging ( $\mathrm{MRI}$ ) activation (Mania, Schizophrenia, Controls groups together, contrast: outcome: win > omission of reward) and time courses (first eigenvariate of the fMRI signal intensity as provided by standard SPM functions) in left and right nucleus accumbens (NAcc) for each of the groups. The map was thresholded at $p<0.001$ and extent threshold corrected at $p<0.05$. Mean-corrected first eigenvariate values were extracted from the left and right NAcc functional ROI for each subject and averaged over groups. The time course data were averaged event-related to depict the mean $\mathrm{fMRI}$ signal and standard error related to the outcome phase (win and omission of reward), in each of the three groups. Grey shades indicate the period when reward expectation and receipt or omission of reward took place relative to the MR signal, assuming a delay of the BOLD signal of $6 \mathrm{~s}$. $\Uparrow$ : significant differences $(p<0.05)$ between conditions. $\Downarrow$ : significantly $(p<0.05)$ greater difference (win $>$ omission of reward) in controls than in mania. (b) Mean values of modeled effects in each of the three groups for the contrast (outcome: win $>$ omission of reward) from the regions of interest (ROI) defined from the NAcc activations of the overall analysis of the three groups together. Mania: bipolar manic patients; Schizo.: schizophrenia/schizoaffective patients: Cont.: healthy control subjects.

ventral striatum activation when an expected reward was omitted compared to receipt of an anticipated reward in healthy controls and schizophrenia patients (Abler et al, 2005; Knutson et al, 2001a). In contrast, the differences in activation of dopaminergic brain areas upon anticipation and receipt of rewards were markedly reduced in medicated manic patients. We will now review the patterns of brain activation during the expectation and the outcome phase of the experiment.

\section{Expectation Phase}

While the whole brain and ROI analyses did not reveal significant group differences during the expectation phase, the difference in the signal time courses for high and not rewarded stimuli was significantly reduced in manic patients. This pattern was not a result of decreased responses to the rewarded stimuli but rather of a comparatively high fMRI signal upon not rewarded conditions. Furthermore, in contrast to patients with schizophrenia and controls, manic patients did not show the expected acceleration of reaction times in the higher rewarded trials (Abler et al, 2005, 2006). These findings can be interpreted as a deficit in distinguishing potentially rewarding, ie behaviorally relevant from less relevant stimuli. All stimuli were equally salient to the bipolar patients eliciting high fMRI signal in all conditions irrespective of the reward to be expected. In healthy 
subjects, such expectation signals are believed to help prepare for upcoming events and support decision making and planning processes. Dysfunctions of expectation signals could therefore help to explain the observation (Murphy et al, 2001) that manic patients tend to make suboptimal, often disadvantageous, decisions.

However, we observed the same pattern of brain activation seen here in our manic patients in a study of healthy subjects who had taken a single dose of antipsychotic medication (Abler et al, 2007). After a single dose of olanzapine, healthy subjects displayed elevated fMRI signal when expecting no rewards and therefore decreased differential activation compared to the scans without medication. Thus, the effect seen in the manic subjects in this study could be a confound of antipsychotic medication. While we cannot rule out such a confounding effect in our manic subjects, the medicated patients with schizophrenia in this study, who were being treated with even higher doses of antipsychotic medication, showed the normal pattern of ventral tegmental area activation during the expectation phase.

A recent study of untreated patients with schizophrenia revealed decreased differential responses of dopaminergic brain areas when expecting a monetary reward (Juckel et al, 2006b), which normalized with atypical antipsychotic medication (Juckel et al, 2006a). Our study confirms a normal functioning of the reward system in schizophrenia patients medicated with atypical neuroleptics, in contrast to patients with mania who show altered activation patterns despite being medicated with atypical neuroleptics.

\section{Outcome}

Suppression of ventral striatal activity when anticipated rewards were not obtained (omission trials) has been interpreted as a prediction error signal in previous fMRI studies (Abler et al, 2005; Knutson et al, 2001a). The findings from neuroimaging studies in humans are consistent with reports of nonhuman primate studies, which show a phasic decrease in dopamine neuron activity at the time when expected rewards fail to appear (Hollerman and Schultz, 1998). This signal coding error in the prediction of reward resembles the teaching signals employed in computational learning models (Sutton and Barto, 1981) and has been interpreted as an important basis of learning. While both, schizophrenia patients and healthy controls, showed lower NAcc activation upon omission than upon receipt of rewards as a potential correlate of such a learning signal, bipolar manic patients did not display a similar reduction in the activation of dopaminergic brain regions. More specifically, the difference in the left NAcc activation when comparing receipt to omission of an expected reward was significantly smaller in manic patients compared to healthy controls.

This deficit in prediction error monitoring is in line with previous findings in bipolar disorder. For example, manic patients make suboptimal decisions in the Cambridge Decision Making Task and this deficit correlates positively with the clinical severity of mania (Murphy et al, 2001). When manic patients performed this task during PET scanning, they showed abnormal brain activation in dorsal anterior cingulate, frontal polar, and right inferior frontal cortical regions, with anterior cingulate activation being predicted by the severity of manic symptoms (Rubinsztein et al, 2001).

Such impaired anterior cingulate cortex activation could be related to our finding of abnormal NAcc activation in mania. Considering that reinforcement learning signals are conveyed to the cingulate cortex by the mesencephalic dopamine system (Holroyd and Coles, 2002), we suggest that dysfunctional NAcc activation during prediction error processing (as found in this study) leads to cortical and subcortical hyperactivity in mania (Caligiuri et al, 2003). Our findings add to the hypothesis that deficits in gating functions as measured by prepulse inhibition in manic patients may be due to impairments in the cortico-striatial circuitry (Perry et al, 2001).

Accordingly, unsuccessful motor inhibition was associated with decreased striatal fMRI activation in pediatric bipolar disorder. A lack of striatal error signals as demonstrated in our study was suggested to account for these motor regulatory deficits and contribute to impulsivity and irritability (Leibenluft et al, 2007) as observed in bipolar patients. Indeed, elevated measures of state and trait impulsivity have been observed in bipolar manic patients (Swann et al, 2003). Impulsivity was suggested to represent a core characteristic of the disorder (Najt et al, 2007) responsible for symptoms like hyperactivation, excitability, and hasty decision making that could be related to striatal dysfunction as demonstrated here.

Cognitive deficits including early information processing, response inhibition, executive functions, and memory are core characteristics of affective disorders (Tavares et al, 2003). Cognitive impairments like problems of sustained attention that are potentially related to striato-cortical dysfunction (Sarter et al, 1999) occur in acute mania (Clark et al, 2001) and extend into remitted state (Clark et al, 2002). While deficits in more standard tests of cognitive functioning (motor function, memory, planning) do not appear to be specific for manic episodes and do not provide a significant means of differentiating between, eg mania and depression (Martinez-Aran et al, 2004; Quraishi and Frangou, 2002), tasks of decision making or response inhibition seem to be suitable to identify impairments specific to manic episodes (Murphy et al, 2001; Murphy et al, 1999).

\section{Limitations}

The significance of our results is limited by the fact that all patients were treated with antipsychotic medication of various types, potentially influencing the dopaminergic reward system. Due to the small number of subjects and the number of different medication regimens, it was not possible to calculate covariation effects rendering the interpretation of the results more difficult. However, we found distinct patterns of brain activation in the study groups treated with medication, the schizophrenia, and bipolar manic patients, respectively. This makes it unlikely that the decreased reward circuitry activation that we observed in bipolar manic patients is entirely due to the confounding effect of medication.

The small number of subjects investigated limits the generalizability of our data. Replications of the experiment are needed to endorse the significance of our results. 
Although we excluded all subjects with a history of substance dependence, we have to consider the history of illicit drug use and the history of cigarette smoking in some of our subjects as confounding effects. We were able to match our patient groups, especially the mania group, with the control group with regard to the history of illegal drug use. Furthermore, while addicts ascribe higher salience values to drugs of addiction at the expense of decreased sensitivity for other salient events like monetary rewards, this is related to an overall decrease in dopamine activity (Volkow et al, 2004). This pattern cannot explain our finding of increased reward circuitry activity upon not rewarded trials in bipolar manic subjects.

\section{Conclusion}

Our investigation provides initial evidence for dysfunctional reward pathways in acute mania. Specifically, our results implicate alterations in the coding of prediction error signals in the ventral striatum in acutely manic patients. This could be the neural basis for deficits in learning and decision making, features frequently seen in acutely manic patients.

\section{DISCLOSURE/CONFLICT OF INTEREST}

The study was supported by a personal grant to Birgit Abler from the DAAD (German Academic Exchange Service) and by grant R01 MH070560 (Stephan Heckers). Henrik Walter has received a grant from the company KCG. Dost Ongur received honoraria from North Shore Medical Center in Salem, MA, and Vanderbilt Medical School in Nashville, TN, for giving talks. He also has a small private practice, where he received compensation from individuals for professional services. Apart from this, the authors (Birgit Abler, Ian Greenhouse, Dost Ongur, Henrik Walter, Stephan Heckers) declare that, except for income received from their primary employers, no financial support or compensation has been received from any individual or corporate entity over the past 3 years for research or professional service and there are no personal financial holdings that could be perceived as constituting a potential conflict of interest.

\section{REFERENCES}

Abler B, Erk S, Walter H (2007). Human reward system activation is modulated by a single dose of olanzapine in healthy subjects in an event-related, double-blind, placebo-controlled fMRI study. Psychopharmacology (Berl) 191: 823-833.

Abler B, Walter H, Erk S (2005). Neural correlates of frustration. Neuroreport 16: 669-672.

Abler B, Walter H, Erk S, Kammerer H, Spitzer M (2006). Prediction error as a linear function of reward probability is coded in human nucleus accumbens. Neuroimage 31: 790-795.

Altshuler L, Bookheimer S, Proenza MA, Townsend J, Sabb F, Firestine A et al (2005). Increased amygdala activation during mania: a functional magnetic resonance imaging study. $A m \mathrm{~J}$ Psychiatry 162: 1211-1213.

American Psychiatric Association (2000). Diagnostic and Statistical Manual of Mental Disorders. Washington, DC.

Baldessarini RJ, Tarazi FI (2001). Drugs and the treatment of psychiatric disorders - psychosis and mania. In: Hardman JG,
Limbird LE, Gilman AG (eds). Goodman and Gilman's The Pharmacological Basis of Therapeutics, 10th edn. McGraw-Hill.

Blumberg HP, Leung HC, Skudlarski P, Lacadie CM, Fredericks CA, Harris BC et al (2003). A functional magnetic resonance imaging study of bipolar disorder: state- and trait-related dysfunction in ventral prefrontal cortices. Arch Gen Psychiatry 60: 601-609.

Brett M, Valabregue R, Poline J-B (2002). Region of interest analysis using an SPM toolbox. Presented at the 8th International Conference on Functional Mapping of the Human Brain, Sendai, Japan. Neuroimage 16.

Caligiuri MP, Brown GG, Meloy MJ, Eberson SC, Kindermann SS, Frank LR et al (2003). An fMRI study of affective state and medication on cortical and subcortical brain regions during motor performance in bipolar disorder. Psychiatry Res 123: 171-182.

Clark L, Iversen SD, Goodwin GM (2001). A neuropsychological investigation of prefrontal cortex involvement in acute mania. Am J Psychiatry 158: 1605-1611.

Clark L, Iversen SD, Goodwin GM (2002). Sustained attention deficit in bipolar disorder. Br J Psychiatry 180: 313-319.

Cloninger CR (1987). A systematic method for clinical description and classification of personality variants. A proposal. Arch Gen Psychiatry 44: 573-588.

Cloninger CR, Svrakic DM (1997). Integrative psychobiological approach to psychiatric assessment and treatment. Psychiatry 60: $120-141$.

Dreher JC, Kohn P, Berman KF (2006). Neural coding of distinct statistical properties of reward information in humans. Cereb Cortex 16: $561-573$.

Farmer A, Lam D, Sahakian B, Roiser J, Burke A, O'Neill N et al (2006). A pilot study of positive mood induction in euthymic bipolar subjects compared with healthy controls. Psychol Med 36: $1213-1218$.

Gorrindo T, Blair RJ, Budhani S, Dickstein DP, Pine DS, Leibenluft E (2005). Deficits on a probabilistic response-reversal task in patients with pediatric bipolar disorder. Am J Psychiatry 162: 1975-1977.

Hollerman JR, Schultz W (1998). Dopamine neurons report an error in the temporal prediction of reward during learning. Nat Neurosci 1: 304-309.

Holroyd CB, Coles MG (2002). The neural basis of human error processing: reinforcement learning, dopamine, and the errorrelated negativity. Psychol Rev 109: 679-709.

Hwang J, Lyoo IK, Dager SR, Friedman SD, Oh JS, Lee JY et al (2006). Basal ganglia shape alterations in bipolar disorder. Am J Psychiatry 163: 276-285.

Johnson SL (2005). Mania and dysregulation in goal pursuit: a review. Clin Psychol Rev 25: 241-262.

Juckel G, Schlagenhauf F, Koslowski M, Filonov D, Wustenberg T, Villringer A et al (2006a). Dysfunction of ventral striatal reward prediction in schizophrenic patients treated with typical, not atypical, neuroleptics. Psychopharmacology (Berl) 187: 222-228.

Juckel G, Schlagenhauf F, Koslowski M, Wustenberg T, Villringer A, Knutson B et al (2006b). Dysfunction of ventral striatal reward prediction in schizophrenia. Neuroimage 29: 409-416.

Knutson B, Adams CM, Fong GW, Hommer D (2001a). Anticipation of increasing monetary reward selectively recruits nucleus accumbens. J Neurosci 21: RC159.

Knutson B, Cooper JC (2005). Functional magnetic resonance imaging of reward prediction. Curr Opin Neurol 18: 411-417.

Knutson B, Fong GW, Adams CM, Varner JL, Hommer D (2001b). Dissociation of reward anticipation and outcome with eventrelated fMRI. Neuroreport 12: 3683-3687.

Leibenluft E, Rich BA, Vinton DT, Nelson EE, Fromm SJ, Berghorst LH et al (2007). Neural circuitry engaged during unsuccessful motor inhibition in pediatric bipolar disorder. Am J Psychiatry 164: $52-60$. 
Martinez-Aran A, Vieta E, Reinares M, Colom F, Torrent C, Sanchez-Moreno J et al (2004). Cognitive function across manic or hypomanic, depressed, and euthymic states in bipolar disorder. Am J Psychiatry 161: 262-270.

Miller R (1993). Striatal dopamine in reward and attention: a system for understanding the symptomatology of acute schizophrenia and mania. Int Rev Neurobiol 35: 161-278.

Murphy FC, Rubinsztein JS, Michael A, Rogers RD, Robbins TW, Paykel ES et al (2001). Decision-making cognition in mania and depression. Psychol Med 31: 679-693.

Murphy FC, Sahakian BJ, Rubinsztein JS, Michael A, Rogers RD, Robbins TW et al (1999). Emotional bias and inhibitory control processes in mania and depression. Psychol Med 29: 1307-1321.

Najt P, Perez J, Sanches M, Peluso MA, Glahn D, Soares JC (2007). Impulsivity and bipolar disorder. Eur Neuropsychopharmacol 17: 313-320.

Nolen WA (1983). Dopamine and mania. The effects of trans- and cis-clopenthixol in a double-blind pilot study. J Affect Disord 5: 91-96.

Perlis RH, Welge JA, Vornik LA, Hirschfeld RM, Keck Jr PE (2006). Atypical antipsychotics in the treatment of mania: a metaanalysis of randomized, placebo-controlled trials. J Clin Psychiatry 67: 509-516.

Perry W, Minassian A, Feifel D, Braff DL (2001). Sensorimotor gating deficits in bipolar disorder patients with acute psychotic mania. Biol Psychiatry 50: 418-424.

Quraishi S, Frangou S (2002). Neuropsychology of bipolar disorder: a review. J Affect Disord 72: 209-226.

Rubinsztein JS, Fletcher PC, Rogers RD, Ho LW, Aigbirhio FI, Paykel ES et al (2001). Decision-making in mania: a PET study. Brain 124: 2550-2563.
Sarter M, Bruno JP, Turchi J (1999). Basal forebrain afferent projections modulating cortical acetylcholine, attention, and implications for neuropsychiatric disorders. Ann NY Acad Sci 877: $368-382$.

Scherk H, Pajonk FG, Leucht S (2007). Second-generation antipsychotic agents in the treatment of acute mania: a systematic review and meta-analysis of randomized controlled trials. Arch Gen Psychiatry 64: 442-455.

Strakowski SM, Adler CM, Holland SK, Mills NP, DelBello MP, Eliassen JC (2005). Abnormal fMRI brain activation in euthymic bipolar disorder patients during a counting Stroop interference task. Am J Psychiatry 162: 1697-1705.

Strakowski SM, DelBello MP, Zimmerman ME, Getz GE, Mills NP, Ret J et al (2002). Ventricular and periventricular structural volumes in first- versus multiple-episode bipolar disorder. Am J Psychiatry 159: 1841-1847.

Sutton RS, Barto AG (1981). Toward a modern theory of adaptive networks: expectation and prediction. Psychol Rev 88: 135-170.

Swann AC, Pazzaglia P, Nicholls A, Dougherty DM, Moeller FG (2003). Impulsivity and phase of illness in bipolar disorder. J Affect Disord 73: 105-111.

Tavares JV, Drevets WC, Sahakian BJ (2003). Cognition in mania and depression. Psychol Med 33: 959-967.

Volkow ND, Fowler JS, Wang GJ (2004). The addicted human brain viewed in the light of imaging studies: brain circuits and treatment strategies. Neuropharmacology 47(Suppl 1): 3-13.

Worsley KJ, Marrett P, Neelin AC, Friston KJ, Evans AC (1996). A unified statistical approach for determining significant signals in images of cerebral activation. Hum Brain Mapp 4: 58-73.

Yurgelun-Todd DA, Ross AJ (2006). Functional magnetic resonance imaging studies in bipolar disorder. CNS Spectr 11: 287-297. 